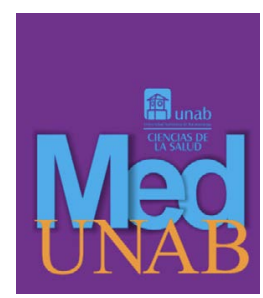

REVISTA DE LA FACULTAD

DE CIENCIAS DE LA SALUD

\title{
Percepción de adolescentes sobre consumo de sustancias psicoactivas en entornos escolares. Estudio cualitativo
}

Adolescents perception of psychoactive substance use in school settings. A qualitative study

Percepção de adolescentes sobre o uso de substâncias psicoativas em ambientes escolares. Estudo qualitativo

Carolina Lucero Enriquez-Guerrero, Enf., MSc. ${ }^{1}$ (D), Yenny Marcela Barreto-Zorza, Enf., MSc. ${ }^{2}$ (D), Lupita Lozano-Vélez, MD. ${ }^{3}$ (D), María Antonia Ocampo-Gómez, MD. ${ }^{4}$ (D)

1. Enfermera, Magister en Enfermería y Epidemiología. Universidad Nacional de Colombia, Bogotá, Colombia.

2. Enfermera, Magister en Salud Pública. Universidad del Rosario, Bogotá, Colombia.

3. Médica. Universidad del Rosario, Bogotá, Colombia.

4. Médica. Universidad del Rosario, Bogotá, Colombia.

Correspondencia. Carolina Lucero Enriquez Guerrero. Universidad Nacional de Colombia Transversal 85G No. 24C-56 Interior 2 Apto 408 Bogotá. Email. clenriquezg@unal.edu.co

\section{INFORMACIÓN DEL ARTÍCULO:}

Artículo recibido: 01 de agosto de 2020

Artículo aceptado: 05 de marzo de 2021

DOI: https://doi.org/10.29375/01237047.3959

Cómo citar. Enriquez-Guerrero CL., Barreto-Zorza YM., Lozano-Vélez L., Ocampo-Gómez MA. Percepción de adolescentes sobre consumo de sustancias psicoactivas en entornos escolares. Estudio cualitativo. MedUNAB. 2021;24(1): 41-50. Doi: https://doi.org/10.29375/01237047.3959

RESUMEN
Introducción. El consumo de sustancias psicoactivas en los adolescentes genera
efectos negativos en el individuo, familia y desarrollo. El objetivo del presente
trabajo es describir la percepción de los adolescentes, sobre los problemas, causas
y consecuencias frente al consumo de sustancias psicoactivas en el entorno escolar.
Metodología. Estudio cualitativo, exploratorio, desarrollo de siete grupos focales.
Muestra por conveniencia hasta saturación de datos. Participaron de manera


voluntaria cuarenta adolescentes de un colegio público de Bogotá, previo consentimiento informado y asentimiento. Guía de 10 preguntas sobre el consumo de psicoactivos. Se hicieron grabaciones de audio y transcripciones, análisis inductivo e interpretativo. Resultados. Se recolectaron datos de adolescentes entre 10 a 13 años de edad. Estos datos se clasificaron en 3 categorías definidas previamente: 1) problema, 2) causas y 3) consecuencias. En la primera emergió una subcategoría; en la segunda y en la tercera, emergieron dos subcategorías. Discusión. Estudios confirman que las causas del consumo de sustancias psicoactivas son maltrato intrafamiliar y bullying, las cuales generan afectaciones a nivel físico y mental. A diferencia de lo reportado en la literatura, los adolescentes no perciben a sus pares y familiares como un factor protector. Conclusión. Desde la percepción de los adolescentes, el acceso al consumo de psicoactivos en entornos escolares es fácil y algunas veces se da por personas externas. Se constituye en un problema conexo con la familia y de las relaciones sociales. Destacan como causas el maltrato intrafamiliar, violencia y bullying. Como consecuencias perciben afectación del desempeño académico, la salud física y psicológica.

Palabras clave:

Adolescente; Trastornos relacionados con sustancias; Acoso escolar; Instituciones académicas; Percepción.

\section{ABSTRACT}

Introduction. The use of psychoactive substances in adolescents generates adverse effects on the individual, family, and development. This study aims to describe the adolescents' perception of the problems, causes, and consequences of psychoactive substance use in the school environment. Methodology. This qualitative, exploratory study involved the development of seven focus groups. Convenience sampling was used until data saturation. Forty adolescents from a public school in Bogota participated voluntarily, with prior informed consent and assent. A 10-question guide on psychoactive drug use was used. Audio recordings and transcripts were subjected to inductive and interpretative analysis. Results. These data were classified into three previously defined categories: 1) problem, 2) causes, and 3) consequences. One subcategory emerged in the first; two subcategories emerged in the second and third. Discussion. Studies confirm that the causes of psychoactive substance use are domestic abuse and bullying, which generate physical and mental effects. Unlike what is reported in the literature, adolescents do not perceive their peers and family members as protecting factors. Conclusion. From the adolescents' perception, access to psychoactive drug use in school environments is easy and sometimes occurs through outsiders. It is a problem related to family and social relations. They highlight as causes intra-family abuse, violence, and bullying. Consequently, they perceive that academic performance, physical and psychological health are affected.

Keywords:

Adolescent; Substance-related disorders.Bullying; Academic institutions; Perception.

\section{RESUMO}

Introdução. O consumo de substâncias psicoativas em adolescentes gera efeitos negativos no indivíduo, na família e no desenvolvimento. O objetivo deste trabalho é descrever a percepção de adolescentes sobre os problemas, causas e consequências do consumo de substâncias psicoativas no ambiente escolar. Metodologia. Estudo qualitativo, exploratório, e desenvolvimento de sete grupos focais. Amostra por conveniência até a saturação dos dados. Quarenta adolescentes de uma escola pública em Bogotá participaram voluntariamente, com consentimento informado previamente e autorização. Guia de 10 questões sobre o consumo de substâncias psicoativas. Foram feitas gravações e transcrições de áudio, análises indutivas e interpretativas. Resultados. Foram coletados dados de adolescentes entre os 10 e 13 anos de idade. Esses dados foram classificados em 3 categorias previamente definidas: 1) problema, 2) causas e 3) consequências. Na primeira, surgiu uma subcategoria; na segunda e terceira emergiram duas subcategorias. Discussão. Estudos confirmam que as causas do consumo de substâncias psicoativas são o abuso doméstico e o 
bullying, que geram afetações físicos e mentais. Diferentemente do que é relatado na literatura, os adolescentes não percebem seus pares e familiares como um fator de proteção. Conclusão. $\mathrm{Na}$ percepção dos adolescentes, o acesso ao uso de substâncias psicoativas em ambientes escolares é fácil e, às vezes, feito por pessoas externas. Torna-se um problema relacionado à família e às relações sociais. Destacam-se como causas o abuso intrafamiliar, violência e bullying. Como consequências, percebem que o desempenho acadêmico, saúde física e psicológica são afetados.

Palavras-chave:

Adolescente; Transtornos relacionados ao uso de substâncias; Assédio escolar; Instituições acadêmicas; Percepção.

\section{Introducción}

Un asunto que genera gran preocupación en el mundo es el relacionado con el uso de las sustancias psicoactivas lícitas e ilícitas entre estudiantes de secundaria, lo que lleva a la necesidad de diseñar intervenciones de promoción y prevención desde la primera infancia (1).

La Organización Mundial de la Salud (OMS) define a la población adolescente como las personas que pertenecen al grupo de edad de 10 a 19 años (2). En este grupo etario se presentan cambios en el comportamiento, percepción y perfiles de riesgo ante el consumo de sustancias psicoactivas (SPA), práctica riesgosa con efectos negativos en su desarrollo $(3,4)$. Una "SPA es aquella sustancia que se consume para alterar la forma en que las personas piensan, se comportan o se sienten, incluyendo tabaco, alcohol y otras sustancias" (5). Es importante establecer la diferencia entre consumo, abuso, y dependencia. Según la Organización Mundial de la Salud (OMS), el abuso de SPA es "un modelo desadaptativo en que el individuo utiliza estas sustancias de manera continua cuando tiene un problema que puede ser social, laboral, psicológico o físico causado por el consumo de esa sustancia en situaciones en las que es físicamente peligroso". Se define como "consumo abusivo un patrón que excede el estándar de consumo moderado o el volumen diario establecido, mientras la dependencia se define como la necesidad de consumir dosis repetidas de SPA para sentirse bien" (6).

Es difícil de identificar cuál tipo de SPA se emplea con más frecuencia en las instalaciones escolares, el colegio sigue siendo un espacio para experimentar e intercambiar nuevas sustancias (1). El consumo de alcohol, cannabis, tabaco y otras SPA ha tenido un incremento exponencial desde la década de los años noventa (2).

De acuerdo con el "Informe sobre el consumo de drogas en las Américas, 2019" (1), se observa una variabilidad en la prevalencia de consumo de alcohol: más del 50\% de los estudiantes de enseñanza secundaria en Argentina,
Chile y Colombia, entre otros, han consumido alcohol durante el último año, convirtiéndose la adolescencia en un período de riesgo crítico para el inicio de su consumo. En cuanto al consumo de tabaco, Colombia tiene una prevalencia de $9,5 \%$ al $15 \%$. El consumo de marihuana es más frecuente entre la población adolescente y adulta joven, con una alta tasa entre los menores de edad, el grupo de 12 a 17 años, el cual supera el $15 \%$ en Canadá y el $10 \%$ en Estados Unidos y Uruguay. En Colombia, este consumo pasó del 7,7\% en 2004 al 8,4\% en 2016. La prevalencia del consumo de cocaína en el último año en Colombia entre los estudiantes de enseñanza secundaria fue de $1,8 \%$ a $3 \%$, datos compartidos con países como Chile y Canadá.

Según un estudio realizado en Bogotá en el año 2016, los adolescentes de 12-17 años son el segundo grupo con mayor prevalencia de consumo de cualquier sustancia ilícita $(7,4 \%)$; en cuanto a estratos socioeconómicos, el mayor consumo se encuentra en el 1, con $6,2 \%$, seguido del 2, con 5,6\%. La marihuana es la más utilizada en Bogotá, y los adolescentes el segundo grupo de mayor consumo $(6,7 \%)$; con respecto al tabaco y el alcohol, los adolescentes en Bogotá representan la menor tasa de utilización $(5,1 \%$ y $13,7 \%$ respectivamente), incrementándose con la edad (7).

La cocaína es la segunda sustancia ilícita más usada en Bogotá, $2,8 \%$ de los escolares del país la han consumido alguna vez en la vida. Se identificó que es más usada en los estudiantes de último año de bachillerato y en colegios privados con relación a los públicos; sin embargo, las diferencias no son estadísticamente significativas. La mayor prevalencia fue en Bogotá y en el Eje Cafetero (8).

Existen otras sustancias importantes, como el consumo de pegantes $(1,8 \%)$ y bazuco $(0,5 \%)$ a escala nacional, siendo Bogotá la tasa más alta de consumo ( $3 \%$ y $0,9 \%$, respectivamente). El éxtasis alcanza el $0,8 \%$ de prevalencia en el país, el consumo de popper y sustancias alucinógenas (LSD), 1,4\% y $1 \%$ respectivamente. A 
pesar de no considerarse una droga ilícita, las bebidas energizantes tienen una alta prevalencia de consumo en este grupo etario, el 19\% refirió haberlas utilizado en el último año (8). Por lo que es importante entender la percepción que tienen los adolescentes sobre los motivos que hacen que una persona se involucre en el consumo de estas sustancias (9).

Estudios indican que "aquellos que empiezan a consumir alcohol o cualquier otra sustancia psicoactiva en las primeras etapas de la adolescencia tienen más probabilidades de desarrollar abuso o dependencia a largo plazo, que aquellos que empiezan a consumir alcohol en la edad adulta" (1), y que con el paso del tiempo, pueda existir mayor riesgo de tener efectos nocivos para la salud.

La utilización de SPA es un problema que no discrimina edad. En la etapa escolar es donde hay una mayor predisposición a consumirlas (5), iniciando su consumo a edades cada vez más tempranas, llegando hasta los 11 o 12 años para el inicio en el alcohol y el tabaco, y uno o dos años después comienzan con marihuana (10).

La percepción es un proceso de formación de representaciones mentales, en que los individuos hacen abstracciones acerca de las cualidades que definen lo esencial de una realidad observada, que se constituye en un flujo informativo constante en tres sentidos: el propio hábitat, las acciones que se efectúan en él y el estado interno de quien percibe. En este proceso participan las emociones que pueden modificar el contenido de la percepción (11). Sin embargo, esto depende del grupo social al que pertenecen los adolescentes, su relación con el medio ambiente y el consumo de sustancias (12). En un estudio efectuado en España, se encontró que los factores relacionados con la experimentación y el abuso de sustancias son de tipo sociocultural y ambiental, la norma social permisiva, aspectos biológicos como el sexo y la genética. La familia, el entorno educativo o el grupo de pares hacen parte de las variables a considerar (13), la dificultad para detectar esta problemática está constituida por las propias peculiaridades de los adolescentes consumidores, caracterizados por la tendencia a la negación y minimización del consumo, la dificultad para aceptar que tienen problemas derivados de este, la presión que reciben de sus iguales para mantenerlo y normalizarlo (14).

Una revisión bibliográfica integrativa sobre la percepción del consumo de drogas en escolares, indica que para ellos estas son dañinas para la salud. Fumar y consumir otra sustancia es sinónimo de "estar loco" y puede contribuir en atacar a los padres, siendo el tabaco la única sustancia que consideran aceptable. También se encontró en esta población que existe una tolerancia social asociada al consumo de drogas por parte de sus familiares. En esta revisión, los escolares perciben que las drogas desestructuran la familia y provocan muerte (15).

Existen varios factores de riesgo identificados para que un adolescente consuma SPA, entre ellos la violencia intrafamiliar, bullying, pobre red de apoyo, entre otros (16). En un estudio sobre patrones de consumo de drogas en adolescentes (4), identificaron que la buena función familiar es un elemento protector para evitar el consumo de SPA eliminando características que vuelven a los adolescentes más vulnerables al consumo. En su estudio evidenciaron que los consumidores de cannabis son los individuos con mayor disfuncionalidad familiar. Otro estudio efectuado en Iraq reporta que la violencia doméstica durante la infancia aumenta la sensación de estrés durante etapas de adultez, lo que lleva a consumir alcohol o SPA (17). Por otro lado, haber sido víctima de bullying se ha asociado con pobre adaptación psicosocial, consumo de alcohol, fumar cigarrillo, ansiedad, depresión, ideas suicidas, desarrollo de trastorno de personalidad antisocial y el consumo de $\operatorname{SPA}(16,18,19)$. Lo anterior, disminuye la autoestima de los adolescentes y los vuelve vulnerables a practicar conductas de alto riesgo, principalmente en menores de 18 años (16). Otro estudio (20), encontró que tener déficit de atención, desorden conductual, de oposición y depresión, son factores de riesgo para consumir SPA.

Se ha descrito un compromiso neurocognitivo en los adolescentes que comienzan el consumo de marihuana y alcohol en comparación con los que lo inician en la edad adulta. Anatómicamente, se ha evidenciado en los cerebros de estas personas una alteración en la sustancia gris y blanca, también se ha descrito una inadecuada y temprana activación neuronal, todo esto relacionado con la presencia de déficit cognitivo (21). Otras consecuencias del consumo de SPA son el insomnio, déficit de atención, procesamiento mental lento, alteraciones en la memoria de trabajo, pobre rendimiento escolar, toma de malas decisiones y pobre inteligencia emocional (21).

Teniendo en cuenta lo anterior, el objetivo de este artículo es describir la percepción de los adolescentes, sobre los problemas, causas y consecuencias frente al consumo de sustancias psicoactivas en el entorno escolar.

\section{Metodología}

Estudio cualitativo, exploratorio, con grupos focales como herramienta cualitativa. Este artículo es producto 
del proyecto "Estrategia de promoción de la salud con enfoque de Atención Primaria en Salud para niños y adolescentes en una localidad de Bogotá", con énfasis general en la Investigación Acción Participación.

La muestra fue por conveniencia hasta lograr la saturación de los datos (22), participaron de manera voluntaria 40 adolescentes. Como criterios de inclusión, se tuvo en cuenta: estudiantes del colegio público, mayores de 11 años, con permanencia mayor a un año en la institución educativa; independientemente de si eran o no consumidores de SPA, dado que no existía un conocimiento previo de esta situación.

El procedimiento para la selección de participantes se realizó en consenso con la institución educativa, tomando las listas de 8 cursos de los grados quintos, sextos y séptimos, que cumplieran los criterios de inclusión. Fueron seleccionados cinco estudiantes de cada curso, a través de una lista de números aleatorios para la participación en los grupos focales.

Estos grupos fueron conducidos mediante un proceso de debate y reflexión, con el fin de lograr una participación activa de los adolescentes (22). El trabajo fue orientado por estudiantes de décimo semestre de Medicina, previamente capacitados, quienes dirigían los grupos focales, con supervisión permanente de los 2 investigadores principales. Se realizaron 7 grupos focales con una guía de preguntas cuyo objetivo fue identificar problemas, causas y consecuencias para el desarrollo del tema. En total fueron planteadas 10 preguntas en forma flexible, que fueron modificadas en función de las respuestas de cada uno de los sujetos. Se pretendió explicar y comprender el fenómeno del consumo de SPA a partir de las percepciones que transmitían los participantes hasta la saturación de datos. Se hicieron grabaciones de audio y transcripciones por parte de los investigadores del estudio, realizando una validación de las mismas.

El análisis de datos se efectuó de acuerdo con los planteamientos de Miles y Huberman (23), en el cual los investigadores del proyecto extrajeron de cada transcripción los descriptores, se elaboró una matriz donde se identificó que los adolescentes perciben las SPA como un problema en su colegio, y se categorizaron los resultados de acuerdo al guion propuesto; posteriormente, en formato Excel se procedió a la codificación de los descriptores que surgieron de la narrativa de los participantes. De acuerdo con el rigor metodológico de la investigación cualitativa, el equipo investigador volvió a campo a confirmar la información con los participantes y se efectuaron los ajustes pertinentes de acuerdo a su percepción (credibilidad), con el apoyo de otros investigadores que analizaron los resultados e interpretación de la información y llegan a hallazgos similares (confirmabilidad). Estos resultados podrían ser trasladados a otras instituciones educativas o grupos con características similares de la ciudad Bogotá (transferabilidad).

\section{Consideraciones éticas}

Se respetaron los principios éticos para las investigaciones médicas en seres humanos de la Declaración de Helsinki (2013) (24). Según lo establecido en la Resolución 8430 de 1993 del Ministerio de Salud de Colombia (25), se garantizó la privacidad de los sujetos de investigación. Esta investigación es considerada de riesgo mínimo. Se obtuvo el aval del Comité de Ética de la Escuela de Medicina y Ciencias de la Salud de la Universidad del Rosario, mediante Acta No. 286 del 7 de mayo de 2015. Se contó con la autorización del colegio de una localidad de Bogotá y el consentimiento informado de los padres de familia y asentimiento de los adolescentes. Los resultados obtenidos fueron presentados en el Colegio objeto de análisis del presente estudio.

\section{Resultados}

\section{Características de los participantes}

La población del estudio estuvo integrada por adolescentes en su mayoría de estratos 1 y 2 de un colegio público ubicado en una zona de estrato 3 de una localidad de Bogotá.

El $60 \%$ son hombres y $40 \%$ son mujeres, todos tenían edades entre 10 y 13 años al momento de hacer la investigación. En cuanto a la antigüedad en la institución, todos los participantes llevaban más de un año estudiando en el colegio, en los grados quinto, sexto y séptimo.

\section{Percepción de adolescentes frente al consumo de SPA}

El consumo de SPA es considerado uno de los principales problemas que se presentan en el colegio y atribuyen la mayor parte a situaciones familiares y sociales. De acuerdo a las 3 categorías predefinidas (problema, causas y consecuencias), se incluyen 5 subcategorías emergentes (Tabla 1). 
Tabla 1. Categorías y subcategorías de la percepción de los adolescentes frente al consumo de Sustancias Psicoactivas. Bogotá 2017

\section{Categoría}

Problema

Causas

\section{Subcategorías}

1. En el colegio y sus alrededores existe fácil acceso a las SPA y algunas veces son compartidas por adultos externos.

2. Los adolescentes perciben que el consumo se presenta por los problemas en casa producidos por violencia intrafamiliar, madres drogadictas y mal ejemplo de los padres o problemas intrainstitucionales como el bullying, decepción amorosa y fracaso estudiantil.

3. Los adolescentes consumen drogas por la influencia de "amistades" o por engaño.

4. El consumo de SPA afecta el rendimiento académico, disminuye la concentración y aumenta el bullying entre estudiantes.

5. Las SPA causan daño en la salud produciendo cáncer, VIH-sida o enfermedades respiratorias y problemas de comportamiento.

Fuente: elaboración propia.

\section{Problema}

\section{Subcategoría 1}

En el colegio, los adolescentes identifican como problemática el consumo de sustancias psicoactivas:

"la droga, el matoneo, las peleas con puños y palabras, que se dan por las diferencias que hay, el bullying y los vicios" (GF3E3A).

Dentro de la institución este consumo se observa en las aulas y las zonas de descanso, en donde los adolescentes buscan lugares apartados, de difícil control por parte de los profesores.

"yo una vez estaba en las escaleras de por allá y estaban intentando compartir droga, cocaína" (GF1E2).

El consumo no se limita a una sola sustancia psicoactiva, sino que existe una gran diversidad de estas, que son consumidas por los adolescentes durante la jornada escolar.

"Marihuana" (GF1E3), "cigarrillo, cocaína y la de una inyección, heroína" (GF1E2), "Ah, o también se ha visto acá el pegante, el bóxer" (GF1E1), "y perico" (GF1E4).

Sin embargo, el consumo de sustancias psicoactivas por parte de los estudiantes no solo se da dentro de la institución sino también en áreas cercanas a esta, donde se encuentran personas que las venden a la hora de descanso y salida de clase de los estudiantes.
“... adultos muy malos que se paran en las rejas y esperan el descanso de los niños para que los niños les compren drogas..." (GF1E1-A).

\section{Causas}

\section{Subcategoría 2}

Los adolescentes identificaron que el consumo de sustancias psicoactivas en el colegio es causa de problemáticas tanto familiares como institucionales. La disfunción familiar es una de las razones que los adolescentes identificaron como causa del consumo.

“... a veces cuando consumen droga es por culpa de los padres, porque los padres dejan la droga digamos... encima de una mesa...” (GF1E1-A); “...Que de pronto la mamá sea drogadicta, que lo maltraten” (GF2E2A).

Esta violencia instaurada dentro de la familia y/o sociedad puede generar que los adolescentes se sientan deprimidos, lo que los lleva al consumo de sustancias para olvidar sus problemas.

“...Puede ser también porque a veces hay personas que, digamos, le matan a la familia y quieren olvidar eso..." (GF1E1-A).

Esto es debido a que las sustancias psicoactivas causan un estado emocional que les ayuda olvidar sus preocupaciones. 
Sin embargo, este consumo también se puede deber a otros problemas del adolescente como alteraciones del estado emocional y su capacidad de generar placer.

“...porque uno se siente triste con personas o algo así por eso es que las consumen para saber si ellos pueden hacer algo, pero no, no pueden...” (GF1E1-A); “... Porque les queda gustando..." (GF2E2A).

Adicionalmente, situaciones como el bullying, fracaso académico y decepciones amorosas, llevan a estos estudiantes a la desesperación o rechazo, lo que tiene como consecuencia el consumo de SPA.

“...le hacen bullying a una persona y la persona está tan desesperada, por ejemplo una niña de mi salón, entonces ella se puede meter drogas, se puede suicidar, algo así..." (GF2E3).

\section{Subcategoría 3}

Alrededor de las instituciones educativas existe venta de SPA, utilizando el engaño como método para inducir su consumo a temprana edad, con estrategias como regalar alimentos, especialmente dulces: “...y también hay unas personas que cuando es Halloween cogen en una envoltura y como que congelan esa droga y la envuelven en ese papel y entonces se lo dan a uno y uno llega a la casa a comer dulces sin saber qué es eso" (GF1E3). Igualmente, aquellos estudiantes que consumen SPA promueven en otros esta conducta, incluso llegando a utilizar métodos de manipulación y presión de grupo, con el propósito de ser aceptados socialmente, lo que es característico de la adolescencia.

“... porque si no lo hacen, pues entonces ya no son sus amigos" (GF3E3A).

\section{Consecuencias}

\section{Subcategoría 4}

Desde la percepción de los adolescentes escolarizados, los estudiantes que consumen SPA son quienes hacen bullying dentro de la institución, generando alteraciones en el comportamiento de los demás estudiantes, además tienden a presentar bajo rendimiento académico debido a que faltan a clases, ya sea evadiendo sus responsabilidades dentro de la institución o mediante el ausentismo escolar. Para ello, se valen de estrategias como la mentira, todo esto basado en la ansiedad de consumo que generan estas sustancias.
“... hay algunos niños que no vuelven al colegio por eso y le dicen mentiras a las mamás para que no los manden al colegio, se hacen los enfermos, se vomitan o los mandan solos al colegio y se van para otra parte..." (GF2E2A).

\section{Subcategoría 5}

Los adolescentes perciben que el consumo de SPA les puede generar perjuicios para su salud física como daños en la piel, cambio de coloración en las uñas, patologías crónicas respiratorias y neurológicas.

“... Pulmonía, enfermedades muy graves como el cáncer o el SIDA. Ataque de epilepsia..." (GF1E1-A).

En lo concerniente al aspecto psicológico, se pueden presentar alteraciones del estado de ánimo, desde la perspectiva de los adolescentes, que pueden conllevar a emociones de placer o tristeza.

“...Las personas se deprimen más... que se ponen felices, son muy bipolares..." (GF3-A).

\section{Discusión}

El consumo de SPA de acuerdo a la percepción de los adolescentes es un problema en el colegio y los alrededores, cuyas causas principales son la disfunción familiar, ser víctima de bullying, problemas académicos y decepciones amorosas. Esto genera como consecuencia para las víctimas el convertirse en personas que realizan bullying, presencia de alteraciones en salud y bajo rendimiento académico.

Los adolescentes perciben que el acceso a las drogas es fácil. En un estudio cualitativo efectuado por Alhyas et al. (26), encontraron que el colegio es el espacio principal para iniciar el consumo de tabaco y otras drogas. El acceso a estas se alcanza a través de amigos, farmacias o en fiestas donde las consiguen gratuitamente; sin embargo, la mayoría de los adolescentes refieren que el territorio escolar es suficiente para comprar las drogas (26).

Como causas del uso de SPA, los adolescentes perciben el bullying, la violencia intrafamiliar, el consumo de los mismos padres y la presión social como los principales motivos que los llevan a consumir. La involucración en bullying y cyberbullying es un gran problema de salud pública debido a las consecuencias en la salud física y mental, haber sido bully o víctima ha tenido asociación con consumo de SPA $(16,18,19)$. 
Así mismo, el bullying es un factor de riesgo para el abuso de SPA debido a que disminuye la autoestima de los adolescentes y los vuelve vulnerables a practicar conductas de alto riesgo como la utilización de SPA, principalmente en menores de 18 años "que sufren acoso escolar, son más vulnerables a consumir drogas, ya que se sienten ignorados, excluidos, discriminados, agredidos..., lo que los hace presa fácil del consumo de dichas sustancias..." $(16,27,28)$. En el estudio efectuado por Siziya et al., encontraron que el consumo de cannabis alguna vez en la vida ha sido asociado con ser víctima del bullying y evidenciaron que consumir esta sustancia los hace más propensos a ser víctimas (29). En un estudio con 78.333 estudiantes de Estados Unidos, encontraron asociación entre ser víctimas y consumo de SPA (30).

La mayoría de los adolescentes del presente estudio perciben la violencia intrafamiliar como otra causa que lleva al consumo, relacionadas con maltrato, muerte violenta y padres consumidores. En el estudio realizado por Horta et al. (31), se encontró que más de un tercio de los estudiantes que consumen SPA han sido testigos de violencia intrafamiliar; el consumo de tabaco fue 2,49 veces mayor en los adolescentes que han presenciado la violencia intrafamiliar, $49 \%$ más alto en los consumidores de alcohol y $60 \%$ mayor en el uso de alguna droga ilícita (31). Otro estudio reporta que casi todos los adolescentes que consumen SPA tienen una mala relación con sus padres (26); la falta de disciplina por parte de los padres se constituye en un factor de riesgo.

En el estudio realizado por Molero-Jurado et al., estos evidenciaron una relación negativa entre la percepción apoyo familiar y la frecuencia en el consumo de tabaco y alcohol, siendo la familia un factor protector para el consumo de estas sustancias; sin embargo, los adolescentes del presente estudio no lo perciben (4). Por otro lado, tener amistades que consuman SPA es el mayor factor de riesgo para el consumo en adolescentes, debido a que el empleo de estas sustancias empieza durante las reuniones grupales; así mismo, rodearse de amistades que no consumen es visto como un factor protector para no abordar conductas (26). Lo anterior no fue percibido por los adolescentes en el presente estudio como un factor de riesgo.

Una revisión sistemática de 32 estudios de cohortes identificados y de los cuales 13 fueron clasificados de alta calidad sobre factores de riesgo para el inicio de su consumo de SPA, reportó que los factores con mayor grado de evidencia fueron, entre otros, tener relaciones problemáticas con parientes y el consumo de la sustancia por parte de los amigos (11).
Como consecuencia del consumo de SPA, los estudiantes del colegio perciben que estas sustancias disminuyen el rendimiento escolar por falta de concentración. El consumo de alcohol antes de los 22 años y su uso excesivo ha demostrado graves efectos a nivel cognitivo como disminución en la atención, memoria de trabajo espacial, memoria verbal y disminución en la velocidad de procesamiento. En el consumo de marihuana antes de los 16-18 años, se han evidenciado cambios neuronales asociados al delta-9-tetrahidrocannabinol con problemas cognitivos más severos en comparación con la edad adulta. Los cambios neurocognitivos son reducción del coeficiente intelectual, memoria verbal, disminución en la atención compleja, lentitud en la velocidad psicomotora y disminución en el funcionamiento neuronal (24).

Sin embargo, el consumo de SPA no solo afecta el ámbito académico sino también la salud física y mental. Según Hall et al., el consumo de cannabis está asociado con aumento de síntomas de bronquitis crónica, frecuencia cardiaca, aparición de síntomas psicóticos o enfermedad psicótica especialmente si presenta antecedente familiar (32). Igualmente, Jouanjous et al., identificaron en 200 pacientes más de 619 efectos adversos producidos por el cannabis, entre ellos desórdenes psiquiátricos, enfermedad nerviosa central y periférica, cardiovascular, respiratoria e intoxicación aguda (33). De forma similar, los adolescentes participantes en el presente estudio perciben que el consumo de SPA les puede generar perjuicios para su salud física tales como daños en la piel, cambio de coloración en las uñas, enfermedades respiratorias y neurológicas. Del mismo modo, a nivel psicológico perciben que pueden presentar alteraciones del estado de ánimo.

Limitaciones: Los resultados se limitan a una sola región colombiana y en el contexto escolar, por lo que es necesario realizar este tipo de estudios en otras regiones del país. Otra limitación fue la no utilización de otras técnicas de recolección de información en investigación cualitativa para lograr la triangulación de los datos.

\section{Conclusiones}

Desde la percepción de los adolescentes, el acceso al consumo de SPA en los entornos escolares es fácil y algunas veces se da por personas externas a la institución educativa. Se constituye en un problema que se da por situaciones relacionadas con la familia y/o sociales y de pares dentro de las que destacan el maltrato intrafamiliar, violencia y bullying. Una vez se desarrolla el consumo, ellos perciben como consecuencias la afectación del desempeño académico, la salud física y psicológica. 
Teniendo en cuenta estos hallazgos, es necesaria la coconstrucción de estrategias de intervención con los adolescentes, sus familias y docentes que permitan fortalecer y empoderar redes de apoyo, generar habilidades de resiliencia para evitar y/o afrontar el consumo de SPA, fortaleciendo las políticas públicas de niñez y adolescencia en un trabajo conjunto con los servicios de salud.

Se recomienda realizar este tipo de investigaciones en otros contextos y otras regiones del país con el fin de generar una comparación entre características y resultados que permita la construcción de lineamientos direccionados a la prevención y manejo del problema del consumo de SPA en adolescentes del país.

\section{Financiación}

Ninguna declarada por los autores.

\section{Conflicto de intereses}

Ninguno declarado por los autores.

\section{Agradecimientos}

Los autores agradecen a los adolescentes participantes, sus familias y al colegio por facilitar la realización de esta investigación.

\section{Referencias}

1. OEA, CICAD. Comisión Interamericana para el Control del Abuso de Drogas. Informe sobre el consumo de drogas en las Américas. [internet]. [citado 04 abr de 2019]. Recuperado a partir de http://cicad. oas.org/Main/ssMain/HTML\%20REPORT\%20 DRUG\%202019/mobile/index.html

2. OMS, OPS. Organización Panamericana de la Salud. Oficina Regional de la Organización Mundial de la Salud. Estrategia y plan de acción regional sobre los adolescentes y jóvenes. [internet]. [citado $04 \mathrm{abr}$ de 2019]. Recuperado a partir de http://new.paho.org/ hq/dmdocuments/2011/Estrategia-y-Plan-de-AccionRegional-sobre-los-Adolescentes-y-Jovenes.pdf

3. Vakkalanka JP, King JD, Holstege CP. Abuse, misuse, and suicidal substance use by children on school property. Clin Toxicol (Phila). 2015;53(9):901-7. https://doi.org/10.3109/15563650.2015.1085998

4. Molero-Jurado MM, Pérez-Fuentes MC, GázquezLinares JJ, Barragán-Martín AB. Análisis y perfiles del consumo de drogas en adolescentes: percepción del apoyo familiar y valoración de consecuencias. Aten Fam. [internet]. [citado 25 may de 2019]; 24(2):56-61. Recuperado a partir de https://www.sciencedirect.com/ science/article/pii/S1405887117300196. h https://doi. org/10.1016/j.af.2017.02.001

5. Fernández Cruz M, Gijón Puerta J, Lizarte Simón E. Consumo de sustancias psicoactivas en estudiantes de magisterio y sus creencias acerca de la educación preventiva. Acción psicol. 2016; 13(1):67-78. https:// doi.org/10.5944/ap.13.1.17419

6. Ministerio de Salud y Protección Social, Oficina de las Naciones Unidas contra la Droga y el Delito (UNODC). Modelo de atención integral para trastornos por uso de sustancias psicoactivas en Colombia. [internet]. [citado 20 may de 2019]. Recuperado a partir de https://www. minsalud.gov.co/sites/rid/Lists/BibliotecaDigital/ RIDE/VS/PP/ENT/modelo-atencion-integralsustancias-psicoactivas-2015.pdf

7. UNODC, CICAD, OEA, Alcaldía Mayor de Bogotá. Estudio de consumo de sustancias psicoactivas en Bogotá, D.C. Informe Final. 2016.

8. UNODC, CICAD, OEA. Estudio Nacional de Consumo de Sustancias Psicoactivas en Población Escolar Colombia-2011. Informe Final. 2011.

9. Vega A, Aramendi P. Las drogas en los centros de iniciación profesional: aportaciones de algunos estudios del país vasco. IPSE-ds. [internet]. [citado 02 de marzo de 2021] 2011:4:57-69. Recuperado a partir de file:///C:/Users/YENNYB 1/AppData/Local/ Temp/Dialnet-LasDrogasEnLosCentrosDeIniciacionP rofesional-3697844.pdf

10. Mendez-Ruiz, MD, De Jesús Alonso-Castillo, MT, Alonso-Castillo, MM, Uribe-Alvarado, J.I, ArmendárizGarcía, NA. Relación de percepción de riesgo y consumo de alcohol en adolescentes. SMAD, Revista Electrónica en Salud Mental, Alcohol y Drogas. [internet]. 2015;11(3):161-167. Recuperado a partir de https:/Www. redalyc.org/articulo.oa?id=80342807007. $\quad$ https://doi. org/10.11606/issn.1806-6976.v11i3p161-167

11. Escobar Montoya JI, Muñoz Astudillo MN, Molina Ceballos NT. Características del fenómeno de las drogas en estudiantes de la Universidad Libre Seccional Pereira. Capítulo 5. Percepciones de los estudiantes acerca del consumo de sustancias psicoactivas. [internet]. 2014 [citado 15 may de 2019]. Recuperado a partir de https://repository. unilibre.edu.co/bitstream/handle/10901/17383/ CARACTER\%C3\%8DSTICAS\%20DROGAS. pdf? sequence $=1 \&$ is Allowed $=$.

12. Baltasar A, Gras MG, Font S, García EM, Patiño $\mathrm{J}$, Raurell M, et al. Creencias de los adolescentes respecto al consumo de drogas. C. Med. Psicosom. [internet]. 2014; 112: 11-21 [citado 02 de mar 2021]; 
(112). Recuperado a partir de https://dialnet.unirioja. es/servlet/articulo?codigo $=4907696$

13. Sánchez JC, Villarreal MH, Ávila MH, Vera A, Musitu G. Contextos de socialización y consumo de drogas ilegales en adolescentes escolarizados, Psychosocial Intervention. 2014;23(1): 69-78. https:// doi.org/10.5093/in2014a7

14. Gamboa AB, Portilla RC. El consumo de drogas como sostén narcisista en la adolescencia. Rev. latinoam. psicopatol. fundam.São Paulo. [internet]. 2018 [citado 02 mar de 2021]; 21(4): 715-738. Recuperado a partir de https://www.sciencedirect.com/science/article/ pii/S1405887117300196. https://doi.org/10.1016/j. af.2017.02.001

15. Fernandes Moll M, Sánchez CharpentierO. Percepción de losniños (as) de 7 a 12 años acerca de las drogasenAmérica Latina. Enfermería Actual de Costa Rica. [internet]. 2015 June [cited Sep 25, 2020]; (28): 83-94. Available from http://www.scielo.sa.cr/scielo.php?script=sci arttext\&pid=S1409-45682015000100083\&lng=en. https://doi.org/10.15517/revenf.v0i28.17047

16. Córdova A, Ramón E, Jiménez K, Cruz C. Bullying y consumo de drogas. Rev. psicol. univ. antioquia, 2011; 4(2):21-48.

17. Lafta R, Al-Shawi A, Al-Nuaimi A, Al Hasnawi S. Impact and relationship of childhood experiences and substance abuse in a population of Baghdad City, Iraq. South East Asia J Public Health. [internet]. 2015 [citado 04 abr de 2019]; 5(1):25-32. Recuperado a partir de https://pdfs.semanticscholar. org/95bb/46c8e6dcbd36175f9900441a76f61508969a. pdf. https://doi.org/10.3329/seajph.v5i1.24848

18. Sigurdson JF, Wallander J, Sund AM. Is involvement in school bullying associated with general health and psychosocial adjustment outcomes in adulthood? Child abuse negl. 2014;38(10):1607-1617. https://doi. org/10.1016/j.chiabu.2014.06.001

19. Kritsotakis G, Papanikolaou M, Androulakis E, Philalithis AE. Associations of bullying and cyberbullying with substance use and sexual risk taking in young adults. J Nurs Scholarsh. 2017;49 (4):360-370. https://doi.org/10.1111/jnu.12299

20. Groenman A, Janssen T, Oosterlaan J. Childhood psychiatric disorders as risk factor for rosubsequent substance abuse: a meta-analysis. J Am Acad Child Adolesc Psychiatry. 2017;56(7):556-569. https://doi. org/10.1016/j.jaac.2017.05.004

21. Lisdahl K, Gilbart E, Wright N, Shollenbarger S. Dare to delay? The impacts of adolescent alcohol and marijuana use onset on cognition, brain structure, and function. Front Psychiatry. 2013;4:53. https://doi. org/10.3389/fpsyt.2013.00053

22. Guber R. El salvaje metropolitano: reconstrucción del conocimiento social en el trabajo de campo. Buenos Aires: Paidós; 2004. P. 111-12.

23. Miles MB, Huberman AM, Saldaña J. Qualitative Data Analysis. 4. ${ }^{\mathrm{a}}$ ed. Los Angeles. United States of America: Sage; 2020.

24. Asociación Médica Mundial. Declaración de Helsinki de la Asociación Médica Mundial. Principios éticos para las investigaciones médicas en seres humanos. Fortaleza: 64.a Asamblea General de la AMM; 2013.

25. Colombia. Ministerio de Salud. Resolución 8430 de 1993. Por la cual se establecen las normas científicas, técnicas y administrativas para la investigación en salud. Bogotá D.C. 1993.

26. Alhyas L, Al Ozaibi N, Elarabi H, El-Kashef A, Wanigaratne S, Almarzouqi A et al. Adolescents' perception of substance use and factors influencing its use: a qualitativestudy in Abu Dhabi. JRSMOpen.2015; 6(2):1-12. https://doi.org/10.1177/2054270414567167

27. Trahtemberg L. DEVIDA drogas y bullying. [internet]. 2009 [citado 20 may de 2019]. Recuperado a partir de http://www.trahtemberg.com/articulos/1450-devidadrogas-y-bullying.html

28. Romaní, F. \& Gutiérrez, C. Auto-reporte de agresividad escolar y factores asociados en escolares peruanos de educación secundaria. Revista Peruana de Epidemiología. [internet] 2011. [citado 02 mar de 2021];15(2):118-125. Recuperado a partir de https:// dialnet.unirioja.es/servlet/articulo?codigo $=3994988$

29. Siziya S, Muula A, Besa Ch, Babaniyi O, Songolo $\mathrm{P}$, Kankiza $\mathrm{N}$ et al. Cannabis use and its sociodemographic correlates among in-school adolescents in Zambia. Ital J Pediatr. 2013; 39 (13): 2-5. https://doi. org/10.1186/1824-7288-39-13

30. Radliff KM, Wheaton JE, Robinson K, Morris $\mathrm{J}$. Illuminating the relationship between bullying and substance use among middle and high school youth. Addict Behav. 2012;37(4):569-72. https://doi. org/10.1016/j.addbeh.2012.01.001

31. Horta Lessa C, Saraiva C, Teixeira V, Welter G, Lessa R. Violence exposure and substance use among southern Brazilian schoolchildren. Psy. Soc. Educ. 2015;7(2):11932. https://doi.org/10.25115/psye.v7i2.528

32. Hall W, Degenhardt L. The adverse health effect of chronic cannabis use. Drug Test Anal. 2014; 6(1-2):3945. https://doi.org/10.1002/dta.1506

33. Jouanjus E, Leymarie F, Tubery M, Lapeyre-Mestre M. Cannabis-related hospitalizations: unexpected serious events identified through hospital databases. Br J Clin Pharmacol. 2011; 71(5):758-65. https://doi. org/10.1111/j.1365-2125.2010.03897.X 